Código JEL: M310

\title{
Aplicación de los Procesos de Identificación Social y Dinámica de los Servicios a las Iniciativas Socialmente Responsables y el Comportamiento del Consumidor Turístico
}

\author{
Patricia MARTÍNEZ GARCÍA DE LEANIZ \\ Universidad de Cantabria \\ martinezrp@unican.es
}

Ignacio RODRÍGUEZ DEL BOSQUE

Universidad de Cantabria

rbosquei@unican.es

\begin{abstract}
RESUMEN
Este estudio profundiza en el efecto que poseen las iniciativas socialmente responsables en el comportamiento del consumidor turístico. Para ello, los autores se basan en dos teorías que han sido identificadas por estudios previos como claves para profundizar en el efecto de la responsabilidad social corporativa en las respuestas comportamentales de diversos grupos de interés de las organizaciones: la Teoría de la Identificación Social y la Teoría de la Dinámica de los Servicios. Para testar su validez se diseñó un modelo conceptual que relaciona la percepción de un comportamiento socialmente responsable por parte de los consumidores con su nivel de satisfacción afectiva y cognitiva, su grado de identificación con la compañía y su nivel de lealtad. Para dar cumplimiento a estos objetivos se llevó a cabo un estudio empírico basado en encuestas. Los resultados muestran como las iniciativas de RSC poseen un efecto positivo y directo sobre la identificación y la satisfacción del consumidor así como un efecto indirecto sobre la lealtad. Igualmente, se demuestra que la identificación C-E posee un efecto positivo y directo sobre la lealtad del consumidor.
\end{abstract}


Palabras clave: Responsabilidad social corporativa, Teoría de la identidad social, Dinámica de los servicios.

Sumario: 1. Introducción, 2. Marco Teórico, 3. Metodología de la investigación, 4. Análisis de Datos, 5. Conclusiones, limitaciones y figuras líneas de investigación.

\title{
Application of Social Identification Processes and Service Dynamics to Corporate Social Responsibility Initiatives and Tourism Consumer Behavior
}

\begin{abstract}
This study explores the effect that socially responsible initiatives have on tourism consumer behavior. The authors based their contribution on two theories that have been identified by previous studies as key to explore the effects of corporate social responsibility on the behavioral responses of various stakeholders toward organizations: the social identification theory and the dynamic of services. To test the validity of this proposal the authors developed a conceptual model relating the perception of a socially responsible behavior by consumers with their level of satisfaction (both affective and cognitive), their degree of identification with the company and level of loyalty. To fulfill these objectives an empirical study based on surveys was conducted. The results show how CSR initiatives have a positive and direct effect on customer identification with the company and customer satisfaction as well as an indirect effect on loyalty. It also shows that their level of identification with the company has a positive and direct effect on consumer loyalty.
\end{abstract}

Keywords: Corporate social responsibility, Social identity theory, Service dynamics.

\section{INTRODUCCIÓN}

La responsabilidad social corporativa (RSC) ha surgido en las últimas décadas no sólo como un importante concepto académico sino también como un imperativo en la agenda corporativa (Mattera y Baena, 2012). Un creciente número de organizaciones desarrollan su actividad adoptando la noción de "socialmente responsables". Una de las principales razones de este creciente interés es debido al efecto de la RSC en el comportamiento del consumidor a la vez que los consumidores exigen a las empresas algo más que productos y servicios de calidad a precio bajo (Bhattacharya y Sen, 2004). Por ello, académicos y profesionales se encuentran cada vez más interesados en analizar cómo reaccionan los consumidores en torno a las iniciativas de RSC. En el ámbito de estudio del comportamiento del consumidor la evidencia empírica sobre la influencia de la 
RSC en el comportamiento del consumidor es aún limitada. La noción de que un comportamiento responsable es una buena práctica de mercado y que genera consecuencias positivas para el negocio es un hecho ampliamente reconocido (García de los Salmones et al., 2005). Sin embargo, aún existe una falta de investigación sobre las consecuencias que poseen las iniciativas de RSC en las respuestas cognitivas y afectivas (creencias, actitudes, atribuciones, identificación, satisfacción), así como comportamentales (lealtad) de los consumidores (Brown y Dacin, 1997; Sen y Bhattacharya, 2003; Klein y Dawar, 2004; Lichtenstein et al., 2004; García de los Salmones et al., 2005; Sen et al., 2006).

En esta línea de investigación, una de las propuestas más innovadoras consiste en considerar que las respuestas del consumidor a las iniciativas de RSC dependen de su grado de identificación con la compañía que realiza dichas actividades (Sen y Bhattacharya, 2001; Lichtenstein et al., 2004; Maignan y Ferrell, 2004; Marin et al., 2009; Currás, 2009). La base para la investigación en torno a la identificación descansa en la Teoría de la Identidad Social (Tajfel y Turner, 1979, 1986). Particularmente, la noción de identificación consumidor-empresa (identificación C-E) se concibe como un estado cognitivo de conexión y proximidad del consumidor con la compañía, generado a través de un proceso (subjetivo) de comparación de los rasgos de su propia personalidad y aquellos de la compañía (Dutton et al., 1994; Bhattacharya y Sen, 2003). Particularmente, en el contexto de la RSC esta línea de investigación se justifica ya que la identificación con la compañía supone una relación significativa para el consumidor que motiva que éste mantenga una unión con la compañía a lo largo del tiempo (Marin et al., 2009). La evolución en la investigación en torno a la RSC aporta resultados alentadores en torno a la relevancia de este constructo en la mejora de la lealtad de los consumidores. Dicho concepto ha sido reconocido durante décadas por su papel en el mantenimiento de exitosos negocios (Dick y Basu, 1994; Oliver, 1999; Chaudhuri y Holbrook, 2001; Lewis y Soureli, 2006). Muy recientemente el constructo RSC ha sido incorporado en modelos clásicos de lealtad. Hasta la fecha, dichas investigaciones se han centrado en los efectos de la RSC y las subsecuentes intenciones de fidelidad (García de los Salmones et al., 2005; Marin et al., 2009; He y Li, 2011).

Ya que la identificación C-E se considera un importante predictor de lealtad (Bhattacharya y Sen 2003; Marin et al., 2009) en el presente estudio se considera igualmente relevante examinar el modo en que esta variable puede ser incorporada en un modelo tradicional de lealtad del consumidor. De este modo, se considera que la influencia de la RSC sobre la lealtad del consumidor se explica en mayor medida a través de la inclusión en el análisis de la influencia de la identificación CE y la satisfacción del consumidor. En este sentido, diversos autores han apuntado a que la inclusión de variables tradicionales y centradas en la explicación de la dinámica de los servicios como la satisfacción, la confianza o el compromiso no han de olvidarse puesto que ayudan a profundizar en los intercambios sociales que 
se producen entre los consumidores y las organizaciones (Harris y Goode, 2004). De este modo, este estudio propone un modelo integrador aunando ambos enfoques teóricos: la Teoría de la Identificación Social y la Dinámica de los Servicios. Tras la revisión de la literatura específica sobre las asociaciones de RSC y la identificación C-E, y sobre el papel que juega la RSC como mecanismo de generación de satisfacción e identificación con la compañía, se propone un modelo teórico de relaciones causales para analizar el efecto indirecto de la RSC sobre la lealtad del consumidor. Además, se describe la metodología empleada y los resultados obtenidos. Finalmente, se presentan las principales conclusiones junto con las implicaciones empresariales, las limitaciones del estudio y las posibles líneas de investigación futuras.

\section{MARCO TEÓRICO}

\section{Asociaciones de RSC e identificación C-E}

Las asociaciones de RSC son una categoría específica de asociaciones corporativas. Brown y Dacin (1997) fueron los primeros autores en acuñar este concepto para categorizar los diferentes tipos de asociaciones cognitivas que los consumidores poseen sobre una marca corporativa. El término asociaciones corporativas se comprende como una etiqueta para aglutinar toda la información sobre la compañía que los consumidores poseen. Las asociaciones corporativas pueden incluir percepciones, inferencias, creencias, cualquier conocimiento sobre el comportamiento de la compañía, información sobre las acciones pasadas de la empresa, los estados de ánimo y las emociones, así como las evaluaciones globales y específicas sobre los distintos atributos de la compañía realizadas por los individuos. Existen diferentes tipos de asociaciones corporativas. Por un lado, las asociaciones de habilidad corporativa - Corporate ability associations- están referidas a la percepción de experiencia y efectividad en la producción de bienes o servicios por parte de la marca. Por su parte, las asociaciones de responsabilidad social corporativa - Corporate social responsability associations-, están referidas al carácter social de la marca, o la voluntad de compromiso social de la misma. Las asociaciones de RSC son un elemento central del concepto global "Asociaciones corporativas", las cuales son distintas de la información que los individuos puedan tener sobre un atributo concreto del producto - Product associations- (Aaker, 1996).

Diversos estudios han analizado el efecto de las asociaciones de RSC sobre el comportamiento del consumidor. Una línea emergente de investigación propone examinar los efectos de las asociaciones de RSC sobre las actitudes y comportamientos del consumidor enfatizando el papel desempeñado por la identificación de este colectivo con la compañía (Currás et al., 2009; Marin et al., 2009; $\mathrm{He}$ y $\mathrm{Li}, 2011)$. A este respecto, diversos autores han identificado la influencia positiva de la RSC sobre la identificación C-E (Lichtenstein et al., 2004; Marin et al., 2009; Currás, 2009; He y Li, 2011). Como ya se ha adelantado, la 
Teoría de la Autocategorización (Turner et al., 1987) y la Teoría de la Identidad Social (Tajfel y Turner, 1979, 1986) han sido utilizadas para explicar las razones y motivos que llevan a los individuos a relacionarse con las compañías. Esto sucede por un proceso cognitivo de categorización donde un individuo se posiciona como miembro de la organización ensalzando las similitudes con otros miembros de la organización y las diferencias con aquellos individuos no miembros. (Turner, 1982). A través de la conciencia de pertenencia y conexión del individuo con la organización el individuo puede lograr una identidad social positiva (Brewer, 1991; Kreiner y Ashforth, 2004). Como resultado de este sentimiento de conexión la organización es aceptada psicológicamente como parte de la identidad personal (Scott y Lane, 2000).

La identificación C-E es un estado cognitivo de auto-categorización, conexión y proximidad del consumidor con la compañía (Bergami y Bagozzi, 2000; Bhattacharya y Sen, 2003), provocado por un proceso (subjetivo) de comparación entre la identidad organizacional y la del propio individuo (Ashforth y Mael, 1989; Dutton et al., 1994). Tal y como Sen y Bhattacharya (2003) señalan, este concepto es sumamente interesante puesto que es el principal sustrato psicológico para construir la clase de relaciones comprometidas, profundas y significativas que los directores de marketing buscan definir hoy en día con sus clientes. En otras palabras, este estado cognitivo de conexión y proximidad implica la percepción de un solapamiento entre la identidad personal y la de la organización (Ashforth y Mael, 1989; Bhattacharya y Sen, 2003), donde la existencia de valores compartidos juega un papel vital (Hunt et al., 1989; Scott y Lane, 2000). La literatura en torno a la identifican organizacional ha demostrado que las iniciativas de RSC revelan una identidad y un carácter que permite a los grupos de interés identificarse con la compañía gracias a la evaluación y valoración del solapamiento entre su propia identidad y la de la compañía (Sen y Bhattacharya, 2001; Maignan y Ferrell, 2004; Lichtenstein et al., 2004). Las iniciativas de RSC asocian la compañía con una imagen positiva de la capacidad de respuesta de la organización a las necesidades de la sociedad (Marin et al., 2009).

Recientes estudios sobre la identificación C-E (Bhattacharya y Sen, 2003, 2004) sugieren que las iniciativas socialmente responsables se configuran como un elemento clave de la identidad corporativa que puede inducir a los consumidores a identificarse y desarrollar un sentimiento de conexión con la organización. De hecho, Lichtenstein et al., (2004) indican que el modo en que las iniciativas de RSC crean beneficios para las compañías parece pasar por el incremento de la identificación con la compañía. De este modo, no sorprende que los consumidores con altos niveles de identificación con la compañía se encuentren más satisfechos (Bhattacharya et al., 1995; Bhattacharya y Sen, 2003; He y Li, 2011). De este modo, los individuos consientes de los esfuerzos de las compañías por desarrollar iniciativas socialmente responsables, desarrollarán niveles de identificación superiores a aquellos individuos que no son conscientes de estas iniciativas (Sen y 
Bhattacharya, 2003). Además, la literatura sugiere que es más probable que los individuos se identifiquen más fácilmente con aquellas compañías con altos niveles de RSC al objeto de incrementar su autoestima y expresar una imagen social más ética (Aquino y Reed II, 2002). De este modo, se plantea la siguiente hipótesis de investigación:

$H_{1}$ : Las asociaciones de RSC tiene un efecto positivo y directo sobre la identificación C-E.

\section{Asociaciones de RSC y satisfacción del consumidor}

Del mismo modo, la literatura académica en el campo del marketing ha sugerido que las asociaciones de RSC pueden influir en la satisfacción del consumidor (Swaen, 2003; Luo y Bhattacharya, 2006; He y Li, 2011; Bigné et al., 2011). Sin embargo, aquellos trabajos en los que se ha testado la relación entre RSC y la satisfacción del consumidor han tendido a basarse en criterios económicos (Luo y Bhattacharya, 2006). Por ello, tal y como recientes investigaciones sugieren, el análisis de la relación entre estos conceptos no basándose en criterios puramente económicos, se configura como una contribución a la literatura académica en el ámbito del marketing (Bigné et al., 2011). Las asociaciones en torno a un comportamiento corporativo responsable crea un contexto favorable en torno a la compañía (y la marca) que favorece la emisión de juicios más favorables sobre la experiencia en torno al servicio o producto (Brown y Dacin, 1997) por lo que los consumidores serán más comprensivos ante fallos en la prestación del servicio mejorando la satisfacción del consumidor. En este sentido, Klein y Dawar (2004) demostraron que una percepción favorable en torno a las asociaciones de RSC ayudan a la causa de un fallo en el servicio a percibirse como inestable, externa a la compañía y por tanto, no controlable por la organización, minimizando las posibles respuestas negativas del consumidor ante fallos en los productos y servicios. De este modo, las asociaciones de RSC pueden influir en la satisfacción del consumidor de las siguientes maneras (Luo y Bhattacharya, 2006). En primer lugar, los consumidores de la compañía pueden formar parte de diversos grupos de interés que se preocupan no sólo del desempeño económico de la compañía sino también del desempeño social de la misma (Maignan et al., 2005). De este modo, es más probable que los consumidores se encuentren más satisfechos con aquellos proveedores de servicios (y productos) que desarrollen iniciativas de RSC y que presente un comportamiento responsable hacia la sociedad (He y Li, 2011). En segundo lugar, las asociaciones de RSC pueden incrementar la utilidad y el valor percibidos, lo que a la vez incrementará la satisfacción del consumidor (Luo y Bhattacharya, 2006). Así, la RSC puede aportar beneficios extra el consumidor, tales como un incremento de la autoestima por lo que estas asociaciones pueden ser un determinante del atractivo de la identidad de la compañía lo que a la vez incrementará la intensidad de la 
identificación del consumidor con la compañía (Marin et al., 2009). De este modo, es más probable que los consumidores con niveles elevados de identificación C-E se encuentren más satisfechos (Bhattacharya et al., 1995; Bhattacharya y Sen, 2003; He y Li, 2011).

Mucha de la investigación en el ámbito del marketing, y específicamente en el área del comportamiento del consumidor en torno al concepto de satisfacción, sugiere una explicación más profunda de este constructo que basando el análisis en términos meramente cognitivos. De hecho, varios estudios sugieren que el origen de la satisfacción del consumidor proviene no exclusivamente del modelo de disconformidad de las expectativas (Oliver, 1977, 1980), sino de otros procesos de naturaleza tanto cognitiva como afectiva (Swan y Trawick, 1993; Oliver, 1997; Cronin et al., 2000). Así, varios académicos apoyan la definición y medición de la satisfacción del consumidor como el resultado de un proceso cognitivo (Oliver, 1977, 1980; Morgan et al., 1996; Kristensen et al., 1999), mientras que por otro lado otra corriente de investigadores comprenden este constructo como resultados de un proceso emocional (Westbrook y Reilly, 1983; Wirtz y Bateson, 1999, Wirtz et al., 2000; Machleit y Mantel, 2001). A este respecto, diversos autores sugieren que las medidas en torno a la satisfacción del consumidor podrían mejorarse utilizando ambas bases teóricas (Westbrook y Reilly, 1983; Bigné y Andreu, 2004). Recientes investigaciones se han basado en los académicos ya mencionados para expresar la satisfacción del consumidor en términos cognitivos y afectivos (Bigné y Andreu, 2004; Bigné et al., 2011). Así, se utiliza esta perspectiva puesto que incluso los autores que inicialmente defendieron el modelo de disconformación de las expectativas como el paradigma clásico en el estudio de esta variable, en la actualidad sugieren la necesidad de considerar nuevas perspectivas en el estudio de este constructo (Westbrook y Oliver, 1991; Hand y Oliver, 1993). Siguiendo esta línea de pensamiento se sugiere que la RSC posee un efecto positivo y directo en la dimensión tanto cognitiva como afectiva de la satisfacción del consumidor. De este modo, se proponen las siguientes hipótesis de investigación:

$\mathrm{H}_{2}$ : Las asociaciones de RSC poseen un efecto positivo y directo sobre la dimensión cognitiva de la satisfacción.

$H_{3}$ : Las asociaciones de RSC poseen un efecto positivo y directo sobre la dimensión afectiva de la satisfacción.

\section{Identificación C-E, satisfacción y lealtad del consumidor}

Además, en este trabajo se propone que el efecto de la identificación C-E sobre la lealtad del consumidor pasa a través de la satisfacción del consumidor por varios motivos. En primer lugar, la literatura académica del marketing ha demostrado ampliamente la influencia de la satisfacción del consumidor sobre la lealtad 
(Biong, 1993; Anderson et al., 1994; Hallowell, 1996; Homburg y Giering, 2001; Bigné y Andreu, 2004). En segundo lugar, la teoría de la disconformación de las expectativas (Oliver, 1977, 1980) propone que es más probable que los consumidores se encuentren satisfechos cuando el actual desempeño de la compañía confirma sus expectativas. La identificación C-E aporta un marco más favorable para que los clientes puedan responder al desempeño de la compañía frente a sus expectativas previas $(\mathrm{He}$ y $\mathrm{Li}, 2011)$. De este modo, cuando las expectativas sobre el desempeño de la compañía se confirman (o exceden), aquellos consumidores altamente identificados con la compañía estarán más satisfechos, puesto que la identificación C-E garantiza a los clientes un vínculo psicológico con la empresa, que a su vez ayuda a los clientes a preservar su autoestima (Aquino y Reed II, 2002). Sin embargo, cuando las expectativas sobre el desempeño corporativo no se confirman, los consumidores altamente identificados con la organización tenderán a estar menos insatisfechos debido a este vínculo afectivo con la compañía (Chaudhuri y Holbrook, 2001). Así, aquellos consumidores altamente identificados son más propensos a encontrarse satisfechos con la compañía (Bhattacharya et al., 1995; Bhattacharya y Sen, 2003; He y Li, 2011). De este modo, se propone:

$\mathrm{H}_{4}$ : La identificación C-E posee un efecto positive y directo sobre la dimensión afectiva de la satisfacción del consumidor.

$H_{5}$ : La identificación C-E posee un efecto positive y directo sobre la dimensión cognitiva de la satisfacción del consumidor.

Investigaciones previas han sugerido que las asociaciones de RSC poseen una influencia en las respuestas del consumidor hacia la compañía y sus productos y servicios. En su estudio, Marin et al. (2009) demostraron que la RSC posee un impacto indirecto sobre la lealtad vía identificación C-E. Sin embargo, ningún estudio previo ha propuesto el efecto mediador de la satisfacción del consumidor sobre los efectos de la RSC en la lealtad del consumidor. Basándonos en la Teoría de la Identificación Social (Tajfel y Turner, 1979, 1986) los consumidores altamente identificados con la compañía son más propensos a participar en actividades a favor de la empresa, tales como proteger su reputación corporativa, apoyar los objetivos de la organización o ser más leales a la misma (Bhattacharya y Sen, 2003). Empíricamente, varios estudios han confirmado estos efectos (Ahearne et al., 2005; Marin et al., 2009; He y Li, 2011). En este sentido, el consumo es la manifestación clave de la relación entre la compañía y sus consumidores.

Aquellos consumidores identificados con la compañía consumirán sus productos como un modo de incrementar su interacción con la firma (Marin et al., 2009; Currás et al., 2009). Estos consumidores tendrán el deseo y el compromiso de contribuir al avance y desarrollo de la organización de la que forman parte 
(Dutton et al., 1994). A través del consumo de los productos y servicios de la compañía los consumidores sentirán que están contribuyendo a los intereses y a los objetivos de la organización (Cornwell y Coote, 2005). Según la Teoría del Yo Extendido (Belk, 1988), la compra de productos y servicios se convierte en un acto de expresión y proyección del yo, necesario para la identificación personal. Así, una vez identificados con la compañía, los consumidores utilizan el consumo de sus productos como un modo de expresar su propia identidad (Yoon et al., 2006). De este modo, un consumidor identificado con la organización será más leal a la misma a través del acto de compra. Por ello, se propone la siguiente cuestión de investigación:

H6: La identificación C-E posee un efecto positivo y directo sobre la lealtad del consumidor.

\section{Satisfacción y lealtad del consumidor}

La lealtad del consumidor es uno de los conceptos más antiguos y que ha despertado mayor interés entre los académicos en el ámbito del marketing. A pesar de que multitud de estudios en torno a este concepto se han desarrollado, el potencial efecto en la relación predictiva existente entre la satisfacción y la futura lealtad del cliente se encuentra aún restringido debido a los resultados ambiguos y contradictorios que existen en la literatura académica (Jacoby y Kyner, 1973; Broyles, 2009). Oliver (1999) define este constructo como un compromiso profunda de recompra de un producto o servicio preferido de manera consistente en el tiempo a pesar de las acciones y esfuerzos de marketing de la competencia, que tienen el potencial de provocar comportamientos de cambio. La literatura académica sugiere que si los consumidores se encuentran satisfechos con las marcas elegidas, estos se sentirán cada vez más inclinados a comprar sus productos y servicios convirtiéndose finalmente en clientes leales. Investigaciones previas han demostrado que la satisfacción del consumidor posee un impacto positivo significativos sobre la retención de los consumidores, el uso de producto y servicio y en la participación se los clientes en las compras de los productos de la compañía (Bolton et al., 2000; Verhoef, 2003), por lo que la satisfacción se convierte en uno de los principales antecedentes de la lealtad del consumidor (Zeithaml y Bitner, 1996; Hu et al., 2009). Sin embargo, multitud de investigadores se cuestionan la fiabilidad de esta afirmación (Oliver, 1999; Reichheld, 2003; Story y Hess, 2006). No es el objetivo del presente estudio analizar este punto en profundidad, sino simplemente determinar si la relación entre estas dos variables existe en el contexto específico de esta investigación. Así, y basándonos en la previa literatura en torno a la satisfacción del consumidor se propone:

$H_{7}$ : La dimensión afectiva de la satisfacción posee un efecto positivo y directo en la lealtad del consumidor. 
$H_{8}$ : La dimensión cognitiva de la satisfacción posee un efecto positivo y directo en la lealtad del consumidor.

Figura 1.

Modelo teórico

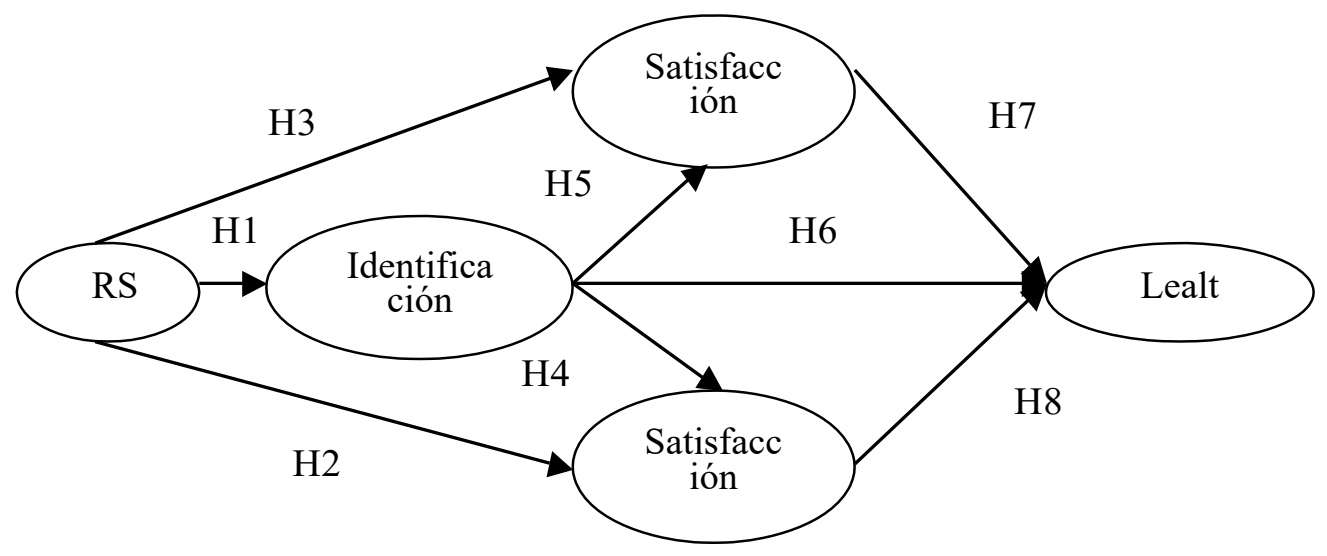

\section{METODOLOGÍA}

\section{Recolección de los datos y diseño de la muestra}

Al objeto de testar las hipótesis de investigación planteadas se realizaron encuestas personales a clientes de establecimientos hoteleros mayores de 18 años en Cantabria de acuerdo a un cuestionario estructurado desarrollado previamente por los investigadores. A los encuestados se les indicó que debían contestar las preguntas refiriéndose a hoteles nacionales donde se hubieran hospedado en sus últimas vacaciones durante el último año. Las encuestas se desarrollaron en el hogar de los encuestados para asegurar que éstos se tomaban el tiempo oportuno para contestar las preguntas de un modo calmado y meditado. Al objeto de diseñar la muestra de la investigación se escogió un procedimiento de muestreo no probabilístico (Trespalacios et al., 2005), en concreto un muestro por conveniencia, ya que no se pudo tener acceso a un censo de los clientes de establecimientos hoteleros mayores de 18 años de la Comunidad Autónoma de Cantabria y no es posible, por tanto, conocer la probabilidad de que cualquier elemento particular de la población sea elegido para la muestra (Trespalacios et al., 2005).

De este modo, con el objetivo de garantizar una mayor representatividad de los datos se recurrió a un muestreo polietápico por cuotas, a partir de la caracterización 
de la población según dos criterios relevantes en la investigación: el sexo y la edad del encuestado, que recoge el Instituto Nacional de Estadística en el Padrón Municipal (2010). El trabajo de campo se realizó en el mes de abril de 2011 y, tras la recogida y depuración de la información, se obtuvo un total de 382 encuestas válidas (Tabla 1). La muestra final consistió en 186 mujeres (49\%) y 196 hombres (51\%); 38 individuos con edad inferior a $25(10 \%) ; 74$ con una edad comprendida entre los 25-34 (19.5\%); 71 entre 35-44 (18.5\%); 76 entre 45-54 (20\%) y 123 con una edad superior a los $55(32.1 \%)$.

\section{Tabla 1}

Ficha técnica de la investigación

\begin{tabular}{|c|c|}
\hline Universo & $\begin{array}{c}\text { Clientes de establecimientos hoteleros } \\
\text { mayores de edad }\end{array}$ \\
\hline Ámbito & España (Comunidad Autónoma de Cantabria) \\
\hline Trabajo de campo & Abril 2011 \\
\hline Muestra & 382 cuestionarios válidos \\
\hline Procedimiento de muestreo & $\begin{array}{c}\text { Muestreo polietápico por cuotas según } \\
\text { criterios: } 1 \text { )sexo y 2) edad }\end{array}$ \\
\hline Procesamiento datos & PASW v. 18.0, EQS v. 6.1 \\
\hline
\end{tabular}

\section{Medidas de las variables}

Una versión preliminar del cuestionario fue administrado a una muestra de conveniencia de 18 consumidores, y los resultados del pre-test se utilizaron para mejorar las medidas y el diseño del mismo cuestionario. Para medir las variables se utilizaron diversas escalas tipo Likert de 7 puntos ya preexistentes en la literatura académica (Tabla 2). Las asociaciones de RSC fueron medidas siguiendo el modelo de Brown y Dacin (1997). La identificación C-E fue medida utilizando una escala de cuatro ítems desarrollada por Mael y Ashforth (1992). En cuanto a las dimensiones afectiva y cognitiva de la satisfacción, fueron medidas a través de una escala de 3 ítems casa una de ellas, aportada por Cronin et al. (2000). Finalmente, la lealtad del consumidor fue medida gracias a una escala de 4 ítems desarrollada por Suárez et al. (2007). 


\section{Tabla 2}

Medida de las variables

\begin{tabular}{|c|c|}
\hline Identificador & Ítem \\
\hline \multicolumn{2}{|l|}{ Asociaciones de RSC } \\
\hline RSC1 & Esta compañía protege el medioambiente \\
\hline $\mathrm{RSC} 2$ & $\begin{array}{l}\text { Esta compañía muestra su compromiso hacia la sociedad } \\
\text { mejorando el bienestar de las comunidades en las que opera }\end{array}$ \\
\hline RSC3 & Esta compañía realiza donaciones a causas sociales \\
\hline \multicolumn{2}{|l|}{ Identificación C-E } \\
\hline ICE1 & Cuando alguien critica a (marca) me lo tomo como un insulto \\
\hline ICE2 & Me preocupa qué piensa la gente sobre (marca) \\
\hline ICE3 & Cuando hablo de (marca) normalmente digo nosotros \\
\hline ICE4 & $\begin{array}{l}\text { Cuando (marca) tiene éxito, siento como si yo también lo } \\
\text { tuviera }\end{array}$ \\
\hline \multicolumn{2}{|l|}{ Satisfacción afectiva } \\
\hline SAF1 & Es agradable alojarse en (marca) \\
\hline SAF2 & Me gusta alojarme en un hotel (marca) \\
\hline SAF3 & Alojarme en un hotel (marca) es estupendo \\
\hline \multicolumn{2}{|l|}{ Satisfacción cognitiva } \\
\hline SCG1 & Alojarme en (marca) fue una decisión inteligente \\
\hline SCG2 & Hice lo correcto al contratar los servicios de (marca) \\
\hline SCG3 & $\begin{array}{l}\text { (Marca) ofrece exactamente lo que necesitaba para mi } \\
\text { alojamiento }\end{array}$ \\
\hline \multicolumn{2}{|r|}{ 政 } \\
\hline LEA1 & $\begin{array}{l}\text { Normalmente escojo esta marca como primera opción a la } \\
\text { hora de elegir un establecimiento hotelero }\end{array}$ \\
\hline LEA2 & $\begin{array}{l}\text { Sería costoso en términos de dinero, tiempo y esfuerzo } \\
\text { finalizar la relación con (marca) }\end{array}$ \\
\hline LEA3 & No cambiaría a otra marca hotelera la próxima vez \\
\hline LEA4 & Recomendaría esta marca a otras personas \\
\hline
\end{tabular}

\section{ANÁLISIS Y RESULTADOS}

Para cumplir los objetivos de este estudio, los autores siguieron el método descrito por Anderson y Gerbing (1988). En primer lugar, la bondad de las propiedades psicométricas de los instrumentos de medida fueron analizadas a través de un Análisis Factorial Confirmatorio (AFC) y en segundo lugar, las relaciones estructurales planteadas entre las variables latentes fueron analizadas a través de un Modelo de Ecuaciones Estructurales (SEM). Ambos, las medidas del modelo y las relaciones causales fueron estimados utilizando en Método de Máxima Verosimilitud Robusto utilizando el programa estadístico EQS v.6 (Bentler, 1995).

\section{Propiedades psicométricas de los instrumentos de medida}

Las propiedades psicométricas de los instrumentos de medida (fiabilidad y validez) fueron evaluados a través de un AFC realizado con la totalidad de los constructos teóricos que aparecen en el marco conceptual propuesto utilizando el 
software estadístico v.6 (Bentler, 1995). Siguiendo a Anderson y Gerbing (1998), la fiabilidad de las escalas propuestas se evaluó a través del coeficiente alfa de Cronbach y a través de un Análisis de la Varianza extraída (AVE) (Hair et al., 2010). Los valores de estos estadísticos exceden los mínimos valores recomendados de 0,7 y 0,5 respectivamente (Hair et al., 2010), lo que confirma la fiabilidad interna del modelo. Adicionalmente, todos los ítems son significativos a un nivel de confianza del $95 \%$ y los coeficientes lambda estandarizados exceden el valor de 0,5 (Steemkamp y van Trijp, 1991), confirmando la validez convergente del modelo. Finalmente, para confirmar la validez discriminante se utilizó el proceso descrito por Anderson y Gerbing (1998), donde se estiman los intervalos de confianza de las correlaciones de los constructos y se comparan con la unidad (Tabla 3). De este modo, la medida del modelo propuesto es correcta. Finalmente, la bondad del ajuste del análisis fue verificado a través del estadístico $\chi^{2}$ (S-B $\chi 2$ ) $(\mathrm{p}<0.05)$ y los índices comparativos de ajuste (Ullman, 1996) NFI y NNFI (Bentler y Bonnett, 1980), IFC (Bentler, 1988), e IFI (Bollen, 1989). Todos los valores de los índices de ajuste son superiores a 0,9 (Bentler, 1992), indicando que el modelo presenta un buen ajuste. Por otra parte, a pesar de que el estadístico $\chi^{2}$ es significativo a un nivel de confianza del 95\% ( $\mathrm{p}<0.05)$, no puede considerarse como un indicador fiable de la bondad del ajuste debido a su sensibilidad para muestras que exceden las 200 unidades (Bollen, 1989). La Tabla 4 muestra los estadísticos mencionados para verificar sus valores y los indicadores de la bondad del ajuste del modelo.

\section{Tabla 3}

Validez discriminante

\begin{tabular}{llllll}
\hline & \multicolumn{1}{c}{ RSC } & \multicolumn{1}{c}{ ICE } & SAF & SCG & LEA \\
\hline RSC & $\mathbf{4 . 7 1 ( 1 . 3 9 )}$ & & & & \\
ICE & {$[0.754 ; 0.879]$} & $\mathbf{5 . 2 5 ( 1 . 4 2 )}$ & & & \\
SAF & {$[0.448 ; 0.570]$} & {$[0.483 ; 0.635]$} & $\mathbf{4 . 3 6 ( 1 . 4 8 )}$ & \\
SCG & {$[0.483 ; 0.622]$} & {$[0.384 ; 0.611]$} & {$[0.735 ; 0.861]$} & $\mathbf{4 . 5 7 ( 1 . 2 3 )}$ & \\
\hline LEA & {$[0.624 ; 0.724]$} & {$[0.478 ; 0.663]$} & {$[0.584 ; 0.697]$} & {$[0.532 ; 0.647] \mathbf{4 . 6 1 ( 1 . 3 7 )}$} \\
\hline
\end{tabular}

La diagonal representa la media y la desviación típica (en paréntesis)

\section{Análisis de las relaciones estructurales e hipótesis planteadas}

La Tabla 5 muestra los coeficientes estandarizados para el análisis de las relaciones estructurales. Tal y como puede observarse los índices de la bondad de ajuste del modelo muestran un buen ajuste por lo que es factible testar las hipótesis planteadas.H1 es aceptada (no puede ser rechazada) $\left(\beta=0.385^{*}\right)$ puesto que las asociaciones de RSC poseen un efecto significativo positivo y directo sobre la identificación C-E. De este modo se confirma que la percepción de un comportamiento responsable es capaz de generar estados de conexión y proximidad por parte del consumidor hacia la compañía tal y como argumentan estudios recientes. $\mathrm{H} 2$ y $\mathrm{H} 3$ predicen que las asociaciones de $\mathrm{RSC}$ poseen un 
efecto positivo y directo sobre las dimensiones cognitiva y afectiva de la satisfacción. H2 $(\beta=0.890 *)$ y H3 $\left(\beta=0.751^{*}\right)$ son aceptadas. Estos resultados confirman resultados de investigaciones previas que analizan la relación entre las asociaciones de RSC y la satisfacción del consumidor basándose en criterios no económicos y teniendo en cuenta ambas dimensiones de la satisfacción (Luo y Bhattacharya, 2006; Bigné et al., 2011). A continuación, analizamos las consecuencias de la identificación C-E. H4 y H5 proponen que la identificación C$\mathrm{E}$ posee un efecto directo sobre ambas dimensiones de la satisfacción del consumidor. H4 $\left(\beta=0.329^{*}\right)$ y H5 $\left(\beta=0.240^{*}\right)$ son aceptadas. Además, H6 predice que la identificación C-E posee un efecto positive y directo sobre la lealtad del consumidor. H6 es aceptada $\left(\beta=0.452^{*}\right)$. En resumen, los efectos significativos y positivos del modelo propuesto confirman que la influencia de la RSC sobre la lealtad del consumidor aparecen mediados no sólo por la identificación C-E sino también por la satisfacción del consumidor. Finalmente H7 y H8 predicen que la satisfacción afectiva y cognitiva de la satisfacción poseen un efecto positivo sobre la lealtad del consumidor. Así, H7 $\left(\beta=0.735^{*}\right)$ y H8 $\left(\beta=0.347^{*}\right)$ son aceptadas. De este modo, el modelo propuesto se valida en su totalidad a la vista de los resultados obtenidos.

\section{Tabla 4}

Análisis factorial confirmatorio (AFC) del modelo final

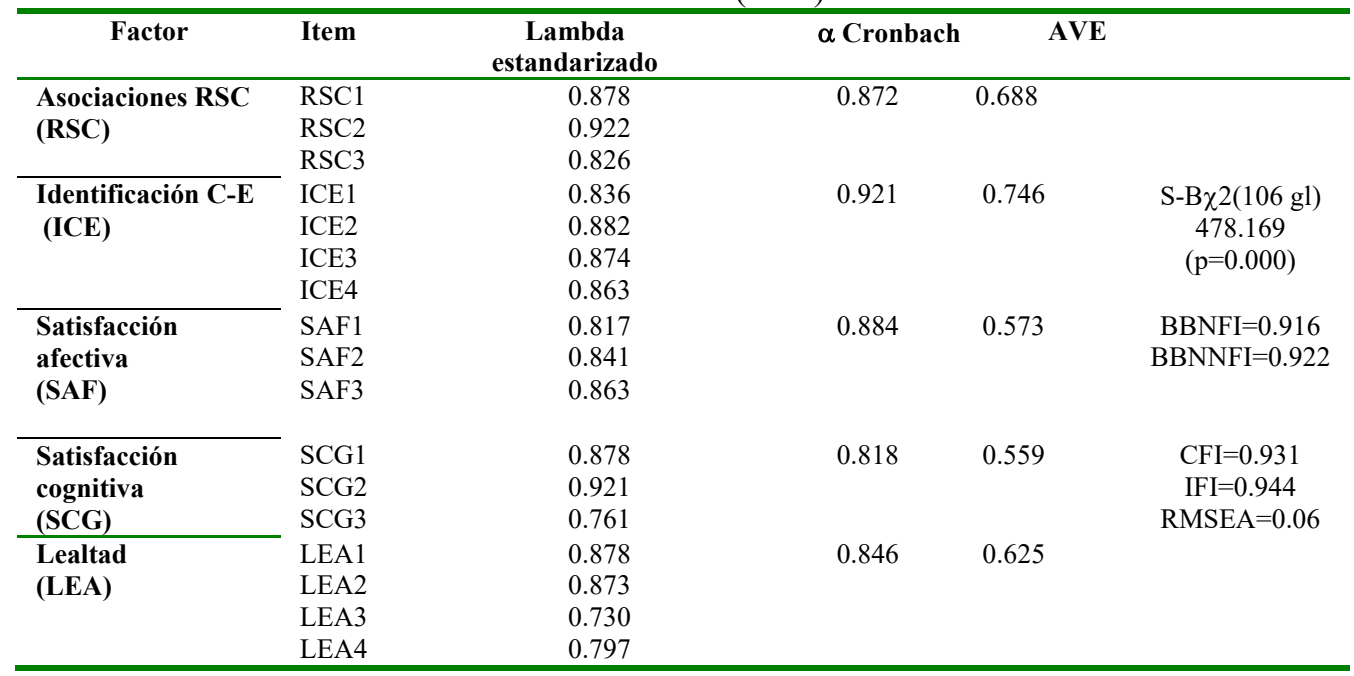


Tabla 5

Resultado del modelo de ecuaciones estructurales

\begin{tabular}{llcl}
\hline Hipótesis & Relación estructural & $\begin{array}{c}\text { Coeficiente estándar (t- } \\
\text { valor robusto) }\end{array}$ & Contraste \\
\hline $\mathrm{H} 1$ & $\mathrm{RSC} \rightarrow$ Identificación C-E & $0.385(3.262)^{*}$ & Aceptado \\
$\mathrm{H} 2$ & $\mathrm{RSC} \rightarrow$ Satisfacción cognitiva & $0.890(11.935)^{*}$ & Aceptado \\
$\mathrm{H} 3$ & $\mathrm{RSC} \rightarrow$ Satisfacción afectiva & $0.751(10.926)^{*}$ & Aceptado \\
$\mathrm{H} 4$ & Identificación C-E $\rightarrow$ Satisfacción cognitiva & $0.329(3.303)^{*}$ & Aceptado \\
$\mathrm{H} 5$ & Identificación C-E $\rightarrow$ Satisfacción afectiva & $0.240(2.895)^{*}$ & Aceptado \\
$\mathrm{H} 6$ & Identificación C-E $\rightarrow$ Lealtad & $0.452(3.931)^{*}$ & Aceptado \\
$\mathrm{H} 7$ & Satisfaccí́n afectiva $\rightarrow$ Lealtad & $0.735(2.874)^{*}$ & Aceptado \\
$\mathrm{H} 8$ & Satisfacción cognitiva $\rightarrow$ Lealtad & $0.347(2.208)^{*}$ & Aceptado \\
\hline \multicolumn{5}{c}{ BBNFI=0.900 BBNNFI=0.917 } & IFI=0.939 RMSEA=0.058 & \\
& CFI $=0.925$ & & \\
\hline
\end{tabular}

$\mathrm{p}<0.05$

Figura 2

Estimación del modelo estructural

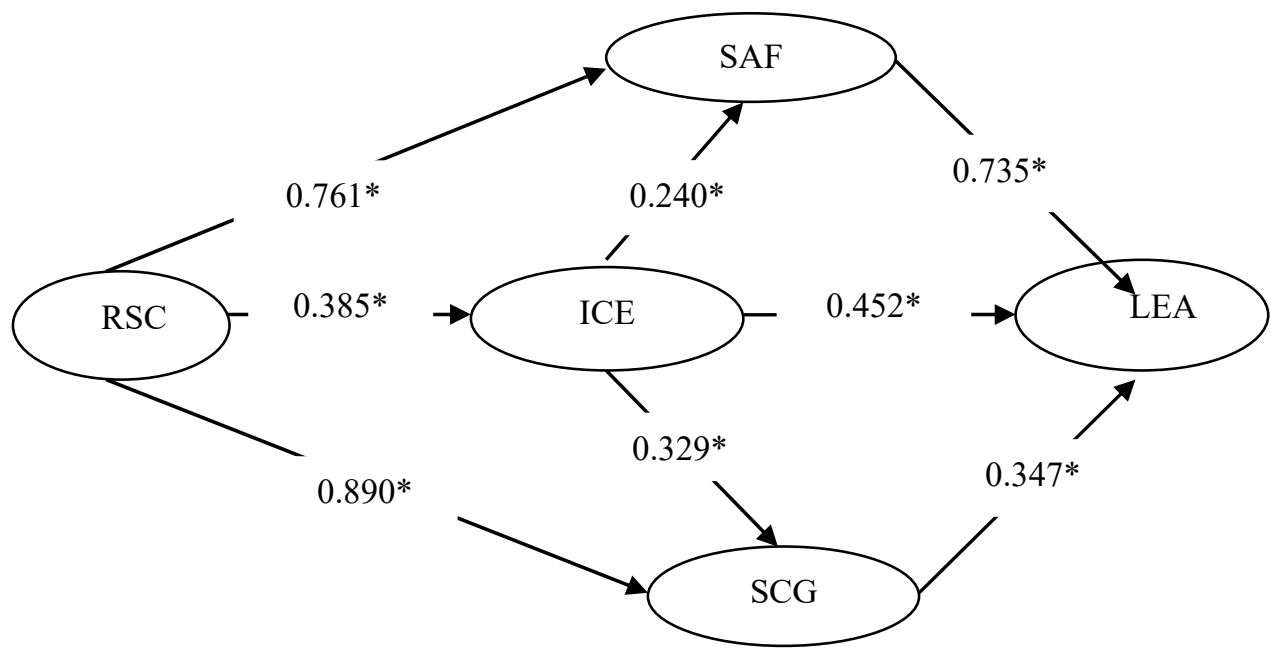

\section{CONCLUSIONES, LIMITACIONES Y FUTURAS LÍNEAS DE INVESTIGACIÓN}

Los objetivos generales de este estudio se basaron en examinar el efecto mediador de la identificación C-E en la influencia de la RSC sobre la lealtad del consumidor. De este modo, se planteó un modelo causal que explica la influencia de las asociaciones de RSC en la identificación C-E y en la satisfacción del consumidor, así como la influencia de la identificación C-E en la lealtad del consumidor. En primer lugar, los resultados confirman que la percepción de un 
comportamiento responsable por parte de las organizaciones es capaz de generar estados de conexión y cercanía por parte del consumidor hacia la compañía (Bhattacharya y Sen, 2003, 2004). Igualmente, este estudio testa y confirma que la identificación C-E juega un papel central en la influencia de las asociaciones de RSC sobre la satisfacción y la lealtad del consumidor. Específicamente, esta investigación expande la visión tradicional existente en la literatura académica sobre el efecto de las asociaciones de RSC sobre el comportamiento del consumidor, y sugiere que este tipo de asociaciones no sólo afectan a las evaluaciones de la compañía y sus productos (Brown y Dacin, 1997; Sen et al., 2006), sino también a la identificación C-E, la satisfacción del consumidor y la lealtad del mismo. Este estudio aporta evidencia empírica adicional a las últimas investigaciones en torno al efecto positivo de la RSC sobre la identificación C-E y la satisfacción del consumidor. A este respecto, este estudio extiende la investigación de Luo y Bhattacharya (2006) proponiendo, desarrollando y confirmando el efecto mediador de la identificación C-E sobre la relación entre la RSC y la satisfacción. Más aún, esta investigación apoya una explicación más completa del constructo satisfacción incluyendo aspectos no meramente cognitivos sino también afectivos. En este sentido, esta investigación permite conceptualizar la satisfacción del consumidor en base a sus dimensiones cognitiva y afectiva tal y como apuntan estudios recientes (Cronin et al., 2000; Wirtz et al., 2000; Machleit y Mantel, 2001).

Consistente con el trabajo de Du et al. (2007), la unión entre la RSC y la lealtad sugiere que las asociaciones de RSC se configuran como un constructo no orientado al corto plazo ni como mecanismo centrado en el incremento de las ventas sino un elemento clave para profundizar en las relaciones a largo plazo con los clientes, a la vista de su efecto en la creación de lealtad a través de la identificación C-E. Este estudio también contribuye a la literatura en torno a la Teoría de la Identidad Social (Tajfel y Turner, 1985) demostrando el papel relevante de la identificación C-E sobre los efectos de la RSC en la satisfacción y lealtad del consumidor. En resumen, los efectos incluidos en nuestro modelo -desde las asociaciones de RSC hasta la lealtad del consumidor- aportan un nuevo enfoque a los gerentes de establecimientos hoteleros en particular, y turísticos en general, en cuento a las posibilidades de las compañías que invierten en iniciativas de RSC.

Todos estos resultados poseen interesantes implicaciones para la gestión de las organizaciones y muestran la existencia de multitud de motivos estratégicos para adoptar iniciativas socialmente responsables. En primer lugar, esta investigación aporta evidencia sobre el hecho de que desarrollando y manteniendo un estado de conexión e identificación entre la compañía y el consumidor es posible generar respuestas positivas hacia la compañía (Bhattacharya y Sen, 2003). De este modo, los gerentes de las organizaciones deberían iniciar y mantener cualquier actividad que beneficie el carácter social de la firma. En este sentido, tal y como argumentan 
recientes investigaciones (García de los Salmones et al., 2005) es importante para las compañías suscribirse a códigos e iniciativas internacionales para formalizar las políticas de RSC y generar dichas ventajas. Además, es necesario destacar que el comportamiento corporativo basado en iniciativas socialmente responsables ha de ser percibido para generar resultados. La conciencia de los consumidores sobre los impactos de la compañía es un pre-requisito para la identificación organizacional (Maignan y Ferrell, 2004). Así, la identificación de los consumidores con la compañía depende del grado en la ésta comunica sus iniciativas de RSC a sus diferentes públicos objetivo. A través de diversos mecanismos como conferencias públicas, anuncios, promociones o newsletters, la comunicación corporativa puede incrementar la imagen de la compañía como buen ciudadano el cual se preocupa por diversas cuestiones que atañen a sus grupos de interés (Scott y Lane, 2000). En segundo lugar, la presente investigación muestra que las asociaciones de RSC incrementan tanto la satisfacción como la lealtad del consumidor, lo que también sugiere la inversión en iniciativas de RSC. Los consumidores tienden a recompensar a aquellas compañías que son más responsables siendo más leales a ellas (Marin et al., 2009; Currás et al., 2009; Currás, 2009).

El significativo papel de la identificación C-E sobre la satisfacción y lealtad del consumidor sugiere que los gerentes deberían invertir en el área de gestión de la identidad corporativa. Como ya se ha adelantado, el estudio de este concepto es un renovado campo de investigación desde que numerosos estudios han demostrado que la gestión eficaz de la identidad corporativa conlleva una generación positiva de la imagen corporativa, y con el tiempo, la mejora de la reputación de la organización, lo que a la vez lleva a los grupos de interés a posicionarse más favorablemente hacia la compañía (Gray y Balmer, 1998; Chun y Davies, 2001; Barnett et al., 2006). Además, la gestión estratégica de la identidad corporativa puede incrementar el sentimiento de pertenencia e identificación entre los grupos de interés (Balmer y Greyser, 2006; He y Balmer, 2007).

A pesar de la contribución de esta investigación, la generalización de los resultados de este estudio es limitada. Este estudio parte de la hipótesis en un contexto de investigación (sector turístico). Por lo tanto, cualquier generalización se encuentra limitada a las circunstancias específicas de este contexto de investigación. Por consiguiente, sería útil reproducir el estudio en otras industrias y sectores para la mejora de la generalización de los resultados. Además, al ser la RSC sensible a las características contextuales (Campbell 2007; Whitehouse 2006) sería interesante replicar este estudio en otros países con una mayor cultura de RSC (p.e. anglosajones). Con respecto a las líneas de investigación futura, sería interesante ampliar este estudio mediante la introducción de las asociaciones de habilidad corporativa ya que este hecho aumentaría la comprensión en torno a cómo se genera la lealtad del cliente. Así mismo, las investigaciones futuras también podrían considerar la inclusión de nuevas variables en el modelo. Con respecto a esto, ya que no existe acuerdo sobre las consecuencias de los efectos de 
la identificación C-E, sería interesante analizar la influencia de este constructo en otras variables afectivas y de comportamiento como las actitudes hacia la empresa y sus productos, o la intención de compra, respectivamente. Si tenemos en cuenta la escasez de estudios orientados hacia los clientes, el presente estudio ha tratado de aportar nuevas ideas acerca de las asociaciones de RSC y sus consecuencias sobre el comportamiento del consumidor. Al mismo tiempo, estas nuevas ideas deberían estimular nuevas investigaciones sobre este tema para la mejora de la comprensión en torno a las implicaciones de la firma en iniciativas socialmente responsables.

\section{BIBLIOGRAFÍA}

AAKER, D.A. (1996), "Measuring brand equity across products and markets", California Management Review, Vol. 38, 102-120.

AHEARNE, M., BHATTACHARYA, C.B. y GRUEN, T. (2005), “Antecedents and consequences of customer-company identification: Expanding the role of relationship management", Journal of Applied Psychology, Vol. 90(3), 574-585.

AQUINO, K. y REED II, A. (2002), "The self-importance of moral identity", Journal of Personality and Social Psychology, Vol. 83(2), 1423-1440.

ANDERSON, E.W., FORNELL, C. y LEHMANN, D.R. (1994), "Customer satisfaction, market share, and profitability: Findings from Sweden", Journal of Marketing, Vol. 58, 53-66.

ANDERSON, J. C. y GERBING, D. W. (1998), "Structural equation modelling in practice: A review and recommended two-step approach", Psychological Bulletin, Vol. 103(3), 411-423.

ASHFORTH, B.E. y MAEL, F. (1989), "Social identity theory and the organization", The Academy of Management Review, Vol. 14(1), 20-39.

BALMER, J.M.T. y GREYSER, S.A. (2006), “Corporate marketing: Integrating corporate identity, corporate branding, corporate communications, corporate image and corporate reputation", European Journal of Marketing, Vol. 40(7), 730-741.

BARNETT, M.L., JERMIER, J.M. y LAFFERTY, B.A. (2006), "Corporate reputation: The definitional landscape", Corporate Reputation Review, Vol. 9, 26-38. 
BELK, R. W. (1988), "Possessions and the extended-self", Journal of Consumer Research, Vol. 15, 139-168.

BENTLER, P.M. y BONNETT, D.G. (1980), "Significance tests and goodness of fit in the analysis of covariance structures", Psychological Bulletin, Vol. 88, 588-606.

BENTLER, P.M. (1988), "Comparative fit indexes in structural models", Psychological Bulletin, Vol. 7, 238-246.

BENTLER, P.M. (1992), "On the fit of models to covariances and methodology to the Bulletin”, Psychological Bulletin, Vol. 112, 400-404.

BENTLER, P. M. (1995), EQS structural equations program manual, Multivariate Software Encino, CA.

BERGAMI, M. y BAGOZZI, R.P. (2000), "Self-categorization, affective commitment and group self-esteem as distinct aspects of social identity in the organization”, British Journal of Social Psychology, Vol. 39, 555-577.

BHATTACHARYA, C.B., RAO, H. y GLYNN, M.A. (1995), "Understanding the bond of identification: An investigation of its correlates among art museum members", Journal of Marketing, Vol. 59, 46-57.

BHATTACHARYA, C.B. y SEN, S. (2003), "Consumer-company identification: A framework for understanding consumers' relationships with companies", Journal of Marketing, Vol. 67(2), 76-88.

BHATTACHARYA, C.B. y SEN, S. (2004), "Doing better at doing good: When, why, and how consumers respond to corporate social initiatives?", California Management Review, Vol. 47, 9-24.

BIGNÉ, J.E. y ANDREU, L. (2004), "Modelo cognitivo-afectivo de la satisfacción en servicios de ocio y turismo", Cuadernos de Economía y Dirección de la Empresa, Vol. 21, 89-120.

BIGNÉ, E., ALVARADO, A., ALDÁS, J. y CURRÁS, R. (2011), "Efectos de la responsabilidad social corporativa percibida por el consumidor sobre el valor y la satisfacción con el servicio", Revista Europea de Dirección y Economía de la Empresa, Vol. 20(4), 139-160.

BIONG, H. (1993), "Satisfaction and loyalty to suppliers within the grocery trade European”, Journal of Marketing, Vol. 27, 21-38. 
BOLTON, R.N, KANNAN, P. K. y BRAMLETT, M. D. (2000), "Implication of loyalty programs and service experiences for customer retention and value", Journal of the Academy of Marketing Science, Vol. 28(1), 95-108.

BREWER, M. B. (1991), "The social self: On being the same and different at the same time", Personality and Social Psychology Bulletin, Vol. 17, 475-482.

BROWN, T.J. y DACIN, P.A. (1997), "The Company and the product: Corporate association and consumer product responses", Journal of Marketing, Vol. $61,68-84$.

BROYLES, S.A. (2009), "Loyalty's influence on satisfaction in cross-cultural settings", Journal of Product \& Brand Management, Vol. 18(6), 414-424.

CAMPBELL, J.L. (2007), "Why would corporations behave in socially responsible ways? An institutional theory of corporate social responsibility", The Academy of Management Review, Vol. 32(3), 946-967.

Instituto Nacional de Estadística (2010), Disponible online en http://www.ine.es.

Chaudhuri, A., \& Holbrook, M.B. (2001), "The chain of effects from brand trust and brand affect to brand performance: The role of brand loyalty", Journal of Marketing, Vol. 65, 81-93.

CHUN, R. y DAVIES, G. (2001), "E-reputation: The role of mission and vision statements in positioning strategy", Brand Management, Vol. 8, 315-33.

CORNWELL, B.T. y COOTE, L.V. (2005), "Corporate sponsorship of a cause: The role of identification in purchase intent", Journal of Business Research, Vol. 58, 268-276.

CRONIN, J.J., BRADY, M.K. y HULT, G.T.M. (2000), “Assessing the effects of quality, value, and customer satisfaction on consumer behavioral intentions in service environments", Journal of Retailing, Vol. 76(2), 193-218.

CURRÁS, R. (2009), "Effects of perceived identity based on corporate social responsibility: The role of consumer identification with the company", Corporate Reputation Review, Vol. 12(2), 177-191.

CURRÁS, R., BIGNÉ, E. y ALVARADO, A. (2009), “The role of self-definitional principles in consumer identification with a socially responsible company", Journal of Business Ethics, Vol. 89(4), 547-564. 
DICK, A. y BASU, K. (1994), "Customer loyalty: Towards an integrated framework", Journal of the Academy of Marketing Science, Vol. 22(2), 99113.

DUTTON, J.E., DUKERICH, J. M. y HARQUAIL, C. V. (1994), “Organizational images and member identification", Administrative Science Quarterly, Vol. 39(2), 239-263.

GARCÍA DE LOS SALMONES, M.M., HERRERO, A. y RODRÍGUEZ DEL BOSQUE, I. (2005), "Influence of corporate social responsibility on loyalty and valuation of services", Journal of Business Ethics, Vol. 61, 369-385.

GRAY, E.R. y BALMER, J.M.T. (1998), "Managing corporate image and corporate reputation", Long Range Planning, Vol. 31(5), 695-702.

HAIR, J.F., BLACK, W.C., BABIN, B.J. y ANDERSON, R.E. (2010), Multivariate Data Analysis, Pearson Prentice-Hall, Upper Saddle River, NJ.

HALLOWELL, R. (1996), "The relationships of customer satisfaction, customer loyalty, and profitability: An empirical study”, International Journal of Service Industry Management, Vol. 7, 27-42.

HARRIS, L. y GOODE, M. (2004), "The four levels of loyalty and the pivotal role of trust: a study of online service dynamics", Journal of Retailing, Vol. 80, 139-158.

HE, H. y BALMER, J.M.T. (2007), Identity studies: Multiple perspectives and implications for corporate-level marketing. European Journal of Marketing, Vol. 41(7), 765-785.

HE, H. y LI, Y. (2011), "CSR and service brand: The mediating effect of brand identification and moderating effect of service quality", Journal of Business Ethics, Vol. 100, 673-688.

HOMBURG, C. y GIERING, A. (2001), "Personal characteristics as moderators of the relationship between customer satisfaction and loyalty: An empirical analysis", Psychology \& Marketing, Vol. 18(1), 43-66.

HU, H.H., KANDAMPULLY, J. y JUWAHEER, T. D. (2009), "Relationships and impacts of service quality, perceived value, customer satisfaction, and image: An empirical study", The Service Industries Journal, Vol. 29(2), 111-125. 
HUNT, S.D., WOOD, V.R. y CHONKO, L.B. (1989), "Corporate ethical values and organizational commitment", Journal of Marketing, Vol. 53, 79-90.

JACOBY, J. y KYNER, D.B. (1973), "Brand loyalty vs. repeat purchasing behavior", Journal of Marketing Research, Vol. 10, 1-9.

KLEIN, J. y DAWAR, N. (2004), « Corporate social responsibility and consumers' attributions and brand evaluations in product-arm crisis", International Journal of Research in Marketing, Vol. 21, 203-217.

KREINER, G.E. y ASHFORTH, B. E. (2004), "Evidence toward an expanded model of organizational identification", Journal of Organizational Behavior, Vol. 25, 1-27.

KRISTENSEN, K., MARTENSEN, A. y GRONHOLDT, L. (1999), "Measuring the impact of buying behavior on customer satisfaction", Total Quality Management, Vol. 10(4/5), 602-614.

LEWIS, B.R. y SOURELI, M. (2006), "The antecedents of consumer loyalty in retail banking”, Journal of Consumer Behavior, Vol. 5(1), 15-31.

LICHTENSTEIN, D.R., DRUMWRIGHT, M.E. y BRAIG, B.M. (2004), “The effect of corporate social responsibility on customer donations to corporatesupported nonprofits", Journal of Marketing, Vol. 68, 16-32.

LUO, X. y BHATTACHARYA, C.B. (2006), "Corporate social responsibility, customer satisfaction, and market value", Journal of Marketing, Vol. 70(4), $1-18$.

MACHLEIT, K.A. y MANTEL, S.P. (2001), "Emotional response and shopping satisfaction. Moderating effects of shopper attributions", Journal of Business Research, Vol. 54, 97-106.

MAEL, F. y ASHFORTH. B. (1992), "Alumni and their alma mater: A partial test of the reformulated model of organizational identification", Journal of Organizational Behavior, Vol. 13, 103-123.

MAIGNAN, I. y FERRELL, O.C. (2004), "Corporate social responsibility and marketing: An integrative framework", Journal of the Academy of Marketing Science, Vol. 32(1), 3-19. 
MAIGNAN, I., FERRELL, O.C. y FERRELL, L. (2005), “A stakeholder model for implementing social responsibility in marketing", European Journal of Marketing, Vol. 39(9/10), 956-977.

MANO, H. y OLIVER, R.L. (1993), "Assessing the dimensionality and structure of the consumption experience: Evaluation, feeling, and satisfaction", Journal of Consumer Research, Vol. 20, 451-466.

MARIN, L., RUIZ, S. y RUBIO, A. (2009), "The role of identity salience in the effects of corporate social responsibility on consumer behavior", Journal of Business Ethics, Vol. 84(1), 65-78.

MATTERA, M. y BARENA, V. (2012), "Corporate reputation and its social responsibility: A comprehensive vision", Cuadernos de Estudios Empresariales, Vol. 22, 129-149.

MORGAN, M.J., ATTAWAY, J.S. y GRIFFIN, M. (1996), "The role of product/service experience in the satisfaction formation process: A test of moderation", Journal of Consumer Satisfaction, Dissatisfaction and Complaining Behavior, Vol. 9, 104-114.

OLIVER, R.L. (1977), "Effect of expectation and disconfirmation on postexposure product evaluations: An alternative interpretation", Journal of Applied Psychology, Vol. 62(8), 480-486.

OLIVER, R.L. (1980), "A cognitive model of the antecedents and consequences of satisfaction decisions", Journal of Marketing Research, Vol. 17(4), 460-469.

OLIVER, R.L. (1997), Satisfaction: A Behavioral Perspective on the Consumer, McGraw-Hill, New York.

OLIVER, R. L. (1999), “Whence consumer loyalty?”, Journal of Marketing, Vol. $63,33-44$.

REICHHELD, F. (2003), "The one number you need to grow", Harvard Business Review, Vol. 81, 46-54.

SEN, S. y BHATTACHARYA, C.B. (2001), "Does doing good always lead to doing better? Consumer reactions to corporate social responsibility", Journal of Marketing Research. Vol. 38(2), 225-243.

SEN, S., BHATTACHARYA, C.B. y KORSCHUN, D. (2006), "The role of corporate social responsibility in strengthening multiple stakeholder 
relationships: A field experiment", Journal of the Academy of Marketing Science, Vol. 34(2), 158-166.

SCOTT, S. G. y LANE, V. R. (2000), "A stakeholder approach to organizational identity”, The Academy of Management Review, Vol. 25(1), 43-62.

STEENKAMP, J. B. y VAN TRIJP, H. C. M. (1991), "The use of LISREL in validating marketing constructs", International Journal of Research in Marketing, Vol. 8(4), 283-299.

STORY, J. y HESS, J. (2006), "Segmenting customer-brand relations: Beyond the personal relationship metaphor", Journal of Consumer Marketing, Vol. 23(7), 406-413.

SUÁREZ, L., VÁZQUEZ, R. y DÍAZ, A.M. (2007), “Análisis de la confianza y el compromiso como variables clave en las relaciones entre los clientes y las agencias de viaje minoristas", Revista de Análisis Turísticos, Vol. 4, 68-87.

SWAEN, V. (2003), "Consumers 'perceptions, evaluations and reactions to CSR activities", IESEG Working Papers.

SWAN, J.E. y TRAWICK, F.J. (1993), “Consumer satisfaction research: 19831992 accomplishments and future directions", Journal of Consumer Satisfaction, Dissatisfaction and Complaining Behavior, Vol. 6, 28-33.

TAJFEL, H. y TURNER, J.C. (1979), An integrative theory of intergroup conflict. En Austin, W. \& Worchel, S. (eds.), The social psychology of intergroup relations. Monterrey, CA: BrooksCole.

TAJFEL, H. yTURNER, J. C. (1986), The social identity theory of inter-group behavior. En S. Worchel \& L. W. Austin (eds.), Psychology of Intergroup Relations, Nelson-Hall, Chicago.

TRESPALACIOS, J. A., VÁZQUEZ, R. y BELLO, L. (2005), Investigación de Mercados, Thomson, Madrid.

TURNER, J.C. (1982), Towards a cognitive redefinition of the social group. In Tajfel, H. (eds.), Social identity and intergroup relations, Cambridge University Press, Cambridge.

TURNER, J.C., HOGG, M.A., OAKES, P.J., REICHER, S.D. y WETHERELL, M.S. (1987), Rediscovering the social group: A self-categorization theory, Blackwell, Oxford. 
ULLMAN, J.D. (1996), Structural Equation Modelling. En Uriel, E. \& Aldás, J. (Eds.) Análisis Multivariante Aplicado, Thomson.

URIEL, E. y ALDÁS, J. (2005), Análisis multivariado aplicado, Thomson, Madrid.

VERHOEF, P. C. (2003), "Understanding the effect of customer relationship management efforts on customer retention and customer share development”, Journal of Marketing, Vol. 67(4), 30-45.

WESTBROOK, R.A. y REILLY, M.D. (1983), "Value-percept disparity: An alternative to the disconfirmation of expectations theory of consumer satisfaction", Advances in Consumer Research, Vol. 10, 256-261.

WESTBROOK, R.A. y OLIVER, R.L. (1991), "The dimensionality of consumption emotion patterns and consumer satisfaction", Journal of Consumer Research, Vol. 18(1), 84-91.

WHITEHOUSE, L. (2006), "Corporate social responsibility: Views from the frontline”, Journal of Business Ethics, Vol. 63(3), 279-296.

WIRTZ, J. y BATESON, J.E. (1999), “Consumer satisfaction with services: Integrating the environment perspective in services marketing into the traditional disconfirmation paradigm", Journal of Business Research, Vol. 44(1), 55-66.

WIRTZ, J., MATTILA, A.S. y TAN, R.L. (2000), “The moderating role of targetarousal on the impact of affect in satisfaction: An examination in the context of service experiences", Journal of Retailing, Vol. 76(3), 347-365.

YI, Y. y LA, S. (2004), "What influences the relationship between customer satisfaction and repurchase intention? Investigating the effects of adjusted expectations and customer loyalty", Psychology \& Marketing, Vol. 21(5), 351-374.

YOON, Y., GÜRHAN-CANLI, Z. y BOZOK, B. (2006), "Drawing inferences about others based on corporate social responsibilities associations", Journal of Academy of Marketing Science, Vol. 34(2), 106-113.

ZEITHAML, V. A. y BITNER, M. J. (1996), Services Marketing, McGraw-Hill, New York. 